ISSN: 2637-773X

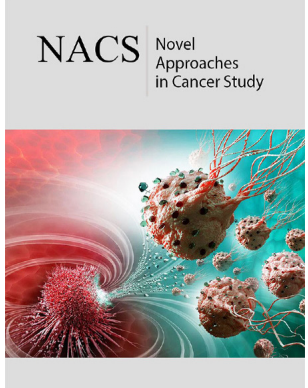

*Corresponding authors: Meser M Ali, Department of Neurosurgery, Cellular and Molecular Imaging Lab, Detroit, USA

Submission: 眥 October 12, 2021

Published: 制October 20, 2021

Volume 6 - Issue 3

How to cite this article: Stephen L Brown James M Snyder, Meser M Ali. DendrimerBased Nanomedicine (Paramagnetic Nanoparticle, Nanocombretastatin, Nanocurcumin) for Glioblastoma Multiforme Imaging and Therapy. Nov Appro in Can Study. 6(3). NACS. 000639. 2021.

DOI: 10.31031/NACS.2021.06.000639

Copyright@ Meser M Ali, This article is distributed under the terms of the Creative Commons Attribution 4.0 International License, which permits unrestricted use and redistribution provided that the original author and source are credited.

\section{Dendrimer-Based Nanomedicine (Paramagnetic Nanoparticle, Nanocombretastatin, Nanocurcumin) for Glioblastoma Multiforme Imaging and Therapy}

\author{
Stephen L Brown, James M Snyder and Meser M Ali* \\ Department of Neurosurgery, USA
}

\begin{abstract}
In brain tumors, delivering nanoparticles across the blood-brain tumor barrier presents a major challenge. Dual mode magnetic resonance imaging and fluorescent imaging probes have been developed where relaxation based Gd-DOTA or ParaCEST agents and a Near-Infrared (NIR) fluorescent dye, DL680 were conjugated on the surface of dendrimer. The in vivo and ex vivo imaging of the dual-modality contrast agent showed excellent potential utility for identifying the location of glioma tumors. Systemic delivery of the subsequent nano-sized agent demonstrated glioma-specific accumulation, probably due to the enhanced permeability and retention effect. The biodistribution studies revealed the G5 agents have accumulated in the glioma tumor and the liver while a G3 agent only accumulated in the brain tumor but not in the liver or kidney. Hydrophobic drug molecules like Combrestatin A4 (CA4) or curcumin have also been conjugated with dendrimers that provided high aqueous solubility with improved therapeutic effect.
\end{abstract}

Keywords: Dendrimer; Nanomedicine; Glioma; Cancer imaging and therapy

\section{Introduction}

Glioblastoma Multiforme (GBM, World Health Organization/ WHO grade IV) is the most common malignant primary brain tumor in adults [1]. GBM is the most common primary tumor of the CNS, and accounts for $12-15 \%$ of all intracranial tumors and $50-60 \%$ of gliomas [2]. GBM is characterized by molecular heterogeneity and the poorest prognosis [2]. Despite the advances made in therapeutic options for GBM, its prognosis remains poor with an overall survival rate ( $<2$ years) that has been stagnant for three decades at approximately $20 \%$. It is one of the unfortunate cancers where no predominant genetic alteration has been identified that could be targeted, resulting in limited therapeutic options. The multimodal treatment of GBM includes maximal surgical resection followed by Radiotherapy (RT) Plus Temozolomide (TMZ) chemotherapy, which may increase median survival to 12-15 months, although the disease typically progresses within 6-9 months, and 2-year survival is less than $25 \%[3,4]$.

Invasive biopsy is routinely utilized to assess histological type, classification, grade and potential aggressiveness of brain cancer and also for determination of the type of drug regimen employed for treatment [5,6]. Imaging techniques include Computed Tomography (CT), Positron Emission Tomography (PET), ultrasound and, most importantly, Magnetic Resonance Imaging (MRI) [7-11]. For some brain tumors the delineation of the actual tumor volume is difficult because peritumoral edema does not readily allow precise discrimination of tumor margins [12]. The use of contrast agent helps overcome this deficiency and allows estimates of tumor margins [13-15]. However, such tumor enhancement using contrast agents is possible only in patients with a compromised Blood-Brain Barrier (BBB) [12]. One approach 
that utilizes the unique structural features of many solid tumors (hypervasculature, defective vascular architecture, and impaired lymphatic drainage) [16,17] may lead to relatively selective extravasation and retention of long circulating nanocarriers. This phenomenon ("passive targeting") is essentially the working principle of most clinically viable targeting strategies based on nanocarriers. This is the Enhanced Permeation and Retention (EPR) effect and has been described for nanoparticulate systems including liposomes, dendrimers, micelles, and polymers [18-20].

Brain tumors demonstrate high levels of angiogenic activity resulting in formation of torturous and abnormally dilated vessels with leaky inter-endothelial gaps and fenestration [2123]. This hyper-permeable vasculature allows nanoparticles to extravasate and be retained in tumor interstitium following systemic administration [24]. Yet, effective transvascular delivery of nanoparticles across the Blood-Brain Tumor Barrier (BBTB) of malignant gliomas remains a challenge. We and others recently developed dendrimer-based paramagnetic nanoparticles that were found to preferentially accumulate in an orthotopic preclinical glioma model with a compromised BBTB [23,25,26]. These dendrimer-based polymers have the advantage of small particle size $[25,26](\sim 7-12 \mathrm{~nm})$ and therefore the potential to improve tumor penetration. Drug molecules can also be conjugated on the surface of dendrimer structure and have the potential for lower systemic toxicity. Hydrophobic anti-cancer drugs like combretastatin a4 (CA4) and Curcumin (Cur) have been conjugated on the surface of a Generation 3 (G3) dendrimer that provide high water solubility and bioavailability with improved therapeutic effects $[27,28]$. Here we review the feasibility of dendrimer- based nanomedicine for glioma imaging and therapy.

\section{Dendrimer-Based Dual Mode Imaging Agent}

Poly (Amidoamine) (PAMAM) dendrimers have been widely used for biomedical applications. This class of polymers has several favorable properties including a well-defined chemical structure, globular shape, low polydispersity index, biocompatibility, and controlled terminal functional groups. Modification of the PAMAM dendrimer surface functional groups with targeting compounds, fluorescent groups, and drugs have produced promising imaging and therapeutic agents [29]. The pharmacokinetics of the dendrimers have been alerted by conjugating non-scaffold polymers such as Polyethylene Glycol (PEG). Oxygen rich PEGs interact with water through hydrogen bonding that minimizes nonspecific interaction with proteins and other biological molecules in the circulation. Moreover, the introduction of PEGs on the surface of dendrimers can reduce the access of the enzymes at the close proximity of the dendrimer conjugates that provides the stability of the loaded drugs/genes from in vivo biodegradation [30].

Intravenous administration of a generation five (G5) PAMAM dendrimer labelled with tritium showed renal-based excretion [31]. Malik et al. [32] reported that I125-labeled PAMAM dendrimer demonstrated $60 \%$ of dendrimer accumulation in the liver and only $1 \%$ of the injected dose remained in the blood circulation one hour after administration [32]. In contrast, introduction of PEGs on the amines surface of PAMAM dendrimer exhibited longer blood half-life [33]. However, dendrimer-based drug delivery cannot be expected to be equally effective across tumor types, sizes, locations, stages and grades. Dendrimers were decorated with relaxationbased MRI contrast agents [24,25,34,35]. Paramagnetic Chemical Exchange Saturation Transfer (PARACEST) agents 23 and Biosensor Imaging of Redundant Deviation in Shifts (BIRDS) agents [36-38].

In a recent study a series of PAMAM dendrimer-based Gn-GdDTPA (G1 to G8) were synthesized, and the pharmacokinetics of the synthesized agents were studied in the BBTB of glioma tumorbearing rats $[24,34,35]$. The properties of dendrimer-based GdDTPA agents in vivo depend on the size, core and exterior surface charge $[39,40]$ and the porosity and pore size of tumor vessels vary with the type and status of the tumor. It was demonstrated that gadolinium chelated dendrimer nanoparticles with core sizes of $<12 \mathrm{~nm}$ permeated the BBTB, whereas larger nanoparticles were hindered [41]; thus, the upper limit of pore size in the BВTВ of malignant brain tumors is approximately $12 \mathrm{~nm}[24,42,43]$. Spherical dendrimer-based paramagnetic nanoparticles ranging between 4 to $10 \mathrm{~nm}$ in diameter maintain peak blood concentrations for several hours [24,41,44]. Gadolinium-based contrast agents have been widely used as contrast media for MRI. But free Gd3+ ions are toxic to biological systems and a suitable ligand or chelate must bind the lanthanide to form a bio-unavailable and nontoxic complex. In 2006, concern associated with the use of $\mathrm{Gd} 3+$ agents were reported due to an apparent link to a disabling condition known as nephrogenic systemic fibrosis (NSF) [45-47]. Two clinically approved agents - omniscan and magnevist - have been associated with NSF [47]. These are all linear Gd3+ chelates based on the structure of Diethylenetriamine Penta-Acetic Acid (DTPA) which is a less thermodynamically stable linear acyclic ligand than macrocyclic chelators. Beyond NSF, safety concerns regarding the retention of Gd3+ in the Central Nervous System (CNS) have intensified after cases of prolonged signal enhancement in the brain were reported, particularly in patients experiencing repeated contrast media administration $[48,49]$. Therefore, much attention has been paid to the development of thermodynamically stable macrocyclic Gd-DOTA based chelates. Recently, a dual mode - MRI and fluorescence agent has been reported where thermodynamically stable macrocyclic Gd-DOTA chelates, and a fluorophore (dylight680) have been conjugated with a Generation 5 (G5) dendrimer (Figure 1) [25]. The pharmacokinetics of GdDOTAG5-DL680 was studied in an experimental rat model of glioma with MRI monitoring [25]. The intravenous delivery of (GdDOTA)54-G5DL680 led to the visualization of the agent in the rat glioma tumor. In another report, a Near Infrared Red (NIR) dye, DyLight680 (DL680) was also conjugated with a dendritic PARACEST (Paramagnetic Chemical Exchange Saturation Transfer) agent to detect glioma in vivo in a compromised BBTB.23 Intravenous delivery of the designed nanomedicine in Figure 1 [(GdDOTA)54G5-DL680], resulted in the agent homing into its glioma tumor site selectively. In vivo MRI detected the agent in a glioma tumor, but not in contralateral tissue. 


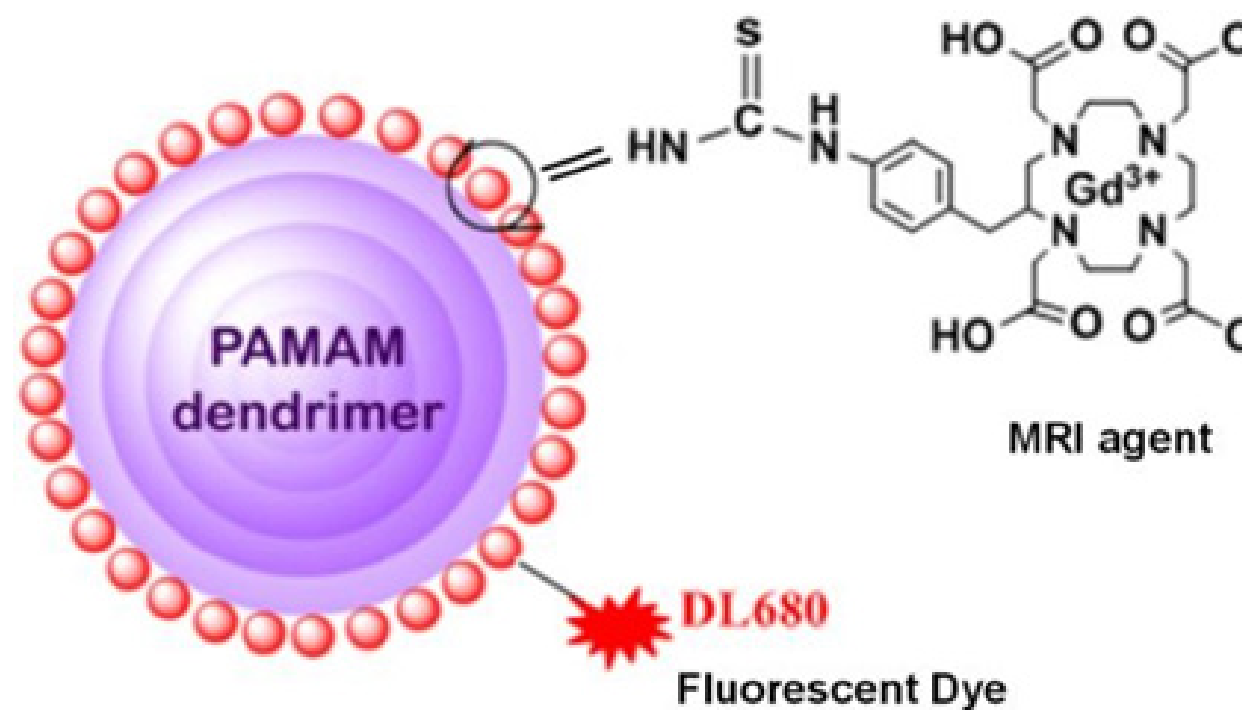

Figure 1: Schematic view of dual-mode dendritic conjugate [(GdDOTA)54-G5- DL680]. MRI contrast agent, GdDOTA is conjugated with a G5 PAMAM dendrimer. A fluorescent dye, DyLight (DL680), is also conjugated with the same dendrimer.

The specificity of the agent was validated by whole-body NIR-optical imaging and ex vivo fluorescence imaging. The in vivo MRI showed the macroscopic location of the tumor while fluorescence imaging showed the biodistribution of the agent. A dual mode MRI-optical approach is ideally suited for in vivo biomedical imaging because MRI provides non-invasive in vivo high-resolution anatomical images, while fluorescence imaging has high sensitivity and can provide microscopic information in ex-vivo pathological tissues. BIRDS is a type of molecular imaging platform for magnetic resonance that utilizes the unique properties of low molecular weight paramagnetic monomers by detecting hyperfine-shifted nonexchangeable protons and transforming the chemical shift information to reflect its microenvironment (e.g., via temperature, $\mathrm{pH}$, etc.). Current BIRDS studies have only used low molecular weight paramagnetic monomers (e.g., Ln-DOTP5-, Ln-DOTMA-, Ln-DOTA-4AmP5-) [36-38]. Despite their capability to accurately report molecular readouts (e.g., temperature, $\mathrm{pH}$, etc.), BIRDS studies with the low molecular weight monomers suffer from short blood half-life and wide in-vivo distribution. To further improve the translational potential of BIRDS, we have conjugated thermodynamically stable macrocyclic p-SCN-Bn-DOTA to the amines on the surface of PAMAM dendrimers from generation 1 (G1) to generation 4 (G4) by using an isothiocyanatobenzyl group that achieves excellent synthesis yield (i.e., greater than $60 \%$ ) and purities [50]. Dendrimer-based BIRDs agents demonstrated both acidic $\mathrm{pH}$ and temperature-sensing properties [50]. Therefore, we hypothesize that dendrimeric BIRDS agents have potential for a range of biomedical applications in quantitative theranostic imaging.

Nanodrug carriers attached to surface ligands or antibodies exploit the receptor-mediated uptake pathways that are recognized by the tumor cells [51-55]. Transferrin receptor-targeting dendrimers have demonstrated efficiency in delivering therapeutic genes and drugs to cancer cells and their use has already led to significant improvements in cancer therapies [56,57].

\section{Dendrimer-Based Drug Conjugate}

The vasculature of GBM is fundamentally different from that of normal vasculature and offers a unique target for anti-cancer therapy. Therefore, direct targeting of tumor vasculature with vascular disrupting agents (VDAs) is distinctly different from antiangiogenic strategies and offers a complementary approach to standard therapies. Combretastatin A4 (CA4) is a potent vascular disrupting drug but insoluble in water. CA4 was conjugated with a G3-succinamic acid PAMAM dendrimer. Conjugation of CA4 with G3 dendrimer improved water solubility as well as bioavailability [28]. However, intravenous (i.v.) delivery of G3-CA4 in an orthotopic glioma model induced necrosis at the core of the tumor leaving a rim of viable tissue. By applying the designed dendrimer-based drug conjugate strategy and tumor-specific prodrug activation mechanism, we observed the true success of inducing necrosis at the core of the tumor in an orthotopic U-251 glioma preclinical animal model [28]. Curcumin (Cur), a yellow pigment in the spice turmeric (curcuma longa), has been reported for its potential chemo preventive and chemotherapeutic activity by influencing various processes, such as cell cycle arrest, differentiation, and apoptosis in a series of cancers [58-65]. In addition, Cur inhibited proliferation, migration and invasion of GBM cells in in-vitro studies [66]. Nevertheless, a major criticism of Cur has been the apparent poor systemic bioavailability in in-vivo animal models. The latter indicates poor relevance for clinical translation even when patients are given up to 8-10grams of the free drug orally each day [63]. In addition, systemic delivery of Cur leads to non-specific distribution throughout the body [67]. It is reasonable to explore novel formulations of Cur that overcome the limitations mentioned above. A Generation 3 (G3) PAMAM dendrimer-based Cur conjugate was 
synthesized [27]. The synthesized G3-Cur conjugate demonstrated full solubility in aqueous media.

The in vitro study revealed that G3-Cur nanoparticles were internalized into glioma U-251 cells. Systemic delivery of G3 Cur conjugate led to preferential accumulation in an orthotopic preclinical glioma model and minimized systemic toxic effects. A dendrimer based G5 dual mode probe accumulated to GBM tumor and liver, respectively [25]. G3-Cur nanoparticles showed more accumulation in tumor and/or less renal uptake, likely due to small particle size, optimal surface charge, and hydrophilicity in blood [27]. Multicolor microscopy images of the tumor tissue showed that G3-Cur particles were internalized inside tumor cells selectively and further localized within nuclei. It has been demonstrated that after oral administration of $400 \mathrm{mg}$ of curcumin to rats only traces of unchanged drug were found in the liver and kidney. At $30 \mathrm{~min}$, $90 \%$ of curcumin was found in the stomach and small intestine, but only $1 \%$ was present at $24 \mathrm{~h} \mathrm{[68].} \mathrm{While} \mathrm{Cur} \mathrm{has} \mathrm{shown} \mathrm{a} \mathrm{wide}$ range of non-specific in vivo distribution, [63]G3-Cur preferentially accumulated at the tumor site. Enhanced bioavailability of G3- Cur conjugate was also observed with improved therapeutic efficacy against cells from different types of cancer. The specificity of G3Cur was further investigated with ex vivo multicolor fluorescence microscopy, which showed accumulation of the particles in glioma tumor tissue selective for nuclear distribution. Ex vivo fluorescence microscopy showed that the G5 agent accumulated in the glioma tumor and the liver [23,25]. Therefore, conjugation of Cur to a smaller size G3 carrier improved bioavailability and tumor targeting [27].

\section{Conclusion}

The overall ineffectiveness of small molecule chemotherapy drugs in treating malignant brain tumors can be attributed to the fact that there is only a transient elevation in drug concentrations within the extravascular extracellular compartments of tumor tissue due to the short blood half-life of small molecule chemotherapy [24]. We and others have developed dendrimer-based paramagnetic nanoparticles that cross the BBTB and accumulate in the GBM tumor site [23,69-71]. Overexpressed cell surface receptors or the process of tumor acidosis are targeted with high affinity ligands. The result demonstrates the relative accumulation of the nanomedicine to the tumor site. The delivery vehicle introduced here can be loaded with imaging agents in combination with a particular drug, thus offering the possibility of developing a nanotheranostic approach to the treatment of GBM.

\section{Acknowledgement}

The authors acknowledge research support from the National Institutes of Health (NIH) grant R01CA206190 to MMA.

\section{References}

1. Johnson DR, O'Neill BP (2012) Glioblastoma survival in the United States before and during the temozolomide era. Journal of Neuro-Oncology 107(2): 359-364

2. Hess KR, Broglio KR, Bondy ML (2004) Adult glioma incidence trends in the United States, 1977-2000. Cancer 101(10): 2293-2299.
3. Wen PY, Kesari S (2008Z) Malignant gliomas in adults. The New England Journal of Medicine 359(5): 492-507.

4. Dhermain F, Ducreux D, Bidault F, Bruna A, Parker F, et al. (2005) Use of the functional imaging modalities in radiation therapy treatment planning in patients with glioblastoma. Bulletin Du Cancer 92(4): 333342 .

5. Becker CM, Beaudry P, Funakoshi T, Benny O, Zaslavsky A, et al. (2011) Circulating endothelial progenitor cells are up- regulated in a mouse model of endometriosis. The American Journal of Pathology 178(4): 1782-1791.

6. Chen AC, Wu MH, Chang CH, Cheng CY, Hsu KY (2011) Single portal endoscopic carpal tunnel release: Modification of Menon's technique and data from 65 cases. International Orthopaedics 35(1): 61-65.

7. Wintermark M, Sincic R, Sridhar D, Chien JD (2008) Cerebral perfusion CT: technique and clinical applications. Journal of Neuroradiology 35(5): 253-260.

8. Tovi M (1993) MR imaging in cerebral gliomas analysis of tumour tissue components. Acta Radiologica Supplementum 384: 1-24.

9. Hanson MW, Glantz MJ, Hoffman JM, Friedman AH, Burger PC, et al. (1991) FDG-PET in the selection of brain lesions for biopsy. Journal of Computer Assisted Tomography 15(5): 796-801.

10. Pichler A, Prior JL, Piwnica-Worms D (2004) Imaging reversal of multidrug resistance in living mice with bioluminescence: MDR1 P-glycoprotein transports coelenterazine. Proceedings of the National Academy of Sciences of the United States of America 101(6): 1702-1707.

11. Boekelheide K, Lee J, Shipp EB, Richburg JH, Li G (1998) Expression of Fas system-related genes in the testis during development and after toxicant exposure. Toxicology Letters 102-103: 503-508.

12. Shamji MF, Fric-Shamji EC, Benoit BG (2009) Brain tumors and epilepsy: pathophysiology of peritumoral changes. Neurosurgical Review 32(3): 275-284.

13. Lehmann P, Vallee JN, Saliou G, Monet P, Bruniau A, et al. (2009) Dynamic contrast-enhanced $\mathrm{T} 2 *$-weighted MR imaging: A peritumoral brain oedema study. Journal of Neuroradiology 36(2): 88-92.

14. Pronin IN, McManus KA, Holodny AI, Peck KK, Kornienko VN (2009) Quantification of dispersion of Gd-DTPA from the initial area of enhancement into the peritumoral zone of edema in brain tumors. Journal of Neuro-Oncology 94(3): 399-408.

15. Schoenegger K, Oberndorfer S, Wuschitz B, Struhal W, Hainfellner J, et al. (2009) Peritumoral edema on MRI at initial diagnosis: An independent prognostic factor for glioblastoma? European Journal of Neurology 16(7): 874- 878.

16. Matsumura Y, Maeda H (1986) A new concept for macromolecular therapeutics in cancer chemotherapy: Mechanism of tumoritropic accumulation of proteins and the antitumor agent smancs. Cancer Research 46(12 Pt 1): 6387-6392.

17. Maeda H, Wu J, Sawa T, Matsumura Y, Hori K (2000) Tumor vascular permeability and the EPR effect in macromolecular therapeutics: A review. Journal of Controlled Release 65(1-2): 271-284.

18. Chang WT, Pan CY, Rajanbabu V, Cheng CW, Chen JY (2011) Tilapia (Oreochromis mossambicus) antimicrobial peptide, hepcidin 1-5, shows antitumor activity in cancer cells. Peptides 32(2): 342-352.

19. Borner MM (2006) Molecular targets in colon cancer. Therapeutische Umschau Revue Therapeutique 63(4): 243-248.

20. Fujisawa H, Koide N, Kono T, Takayama K, Tsukioka K, et al. (2002) Expression of basic fibroblast growth factor and its receptor-1 in cardiac myxoma. The Journal of Cardiovascular Surgery 43(5): 589-594.

21. Veiseh O, Sun C, Fang C, Narayan B, Gunn J, et al. (2009) Specific targeting of brain tumors with an optical/magnetic resonance imaging nanoprobe across the blood-brain barrier. Cancer Research 69(15): 6200-6207. 
22. Jain R, Ellika SK, Scarpace L, Schultz LR, Rock JP, et al. (2008) Quantitative estimation of permeability surface-area product in astroglial brain tumors using perfusion CT and correlation with histopathologic grade. AJNR American Journal of Neuroradiology 29(4): 694-700.

23. Akcay C, Tom ME, Campbell SE, Beecher MD (2013) Song type matching is an honest early threat signal in a hierarchical animal communication system. Proceedings Biological sciences / The Royal Society 280(1756): 20122517.

24. Sarin H, Kanevsky AS, Wu H, Kyle RB, Fung SH, et al. (2008) Effective transvascular delivery of nanoparticles across the blood-brain tumor barrier into malignant glioma cells. Journal of Translational Medicine 6: 80 .

25. Karki K, Ewing JR, Ali MM (2016) Targeting glioma with a dual mode optical and paramagnetic nanoprobe across the blood-brain tumor barrier. J Nanomed Nanotechnol 7(4): 395.

26. Sarin H (2010) On the future development of optimally sized lipid-insoluble systemic therapies for CNS solid tumors and other neuropathologies. Recent Patents on CNS Drug Discovery 5(3): 239-252.

27. Gamage NH, Jing L, Worsham MJ, Ali MM (2016) Targeted theranostic approach for glioma using dendrimer-based curcumin nanoparticle. J Nanomed Nanotechnol 7(4): 393.

28. Gonawala S, Aryal M, Ewing JR, deCarvalho AC, Kalkanis S, et al (2018) MRI monitoring of cerebral blood flow after the delivery of nanocombretastatin across the blood brain tumor barrier. J Nanomed Nanotechnol 9(5): 516

29. Luong D, Kesharwani P, Deshmukh R, Cairul M, Umesh G, et al. (2016) PEGylated PAMAM dendrimers: Enhancing efficacy and mitigating toxicity for effective anticancer drug and gene delivery. Acta Biomaterialia 43: 14-29.

30. Kaminskas LM, Boyd BJ, Porter CJ (2011) Dendrimer pharmacokinetics: the effect of size, structure and surface characteristics on ADME properties. Nanomedicine (Lond) 6(6): 1063-1084.

31. Kukowska-Latallo JF, Candido KA, Cao Z, Shraddha N, Istvan M, et al. (2005) Nanoparticle targeting of anticancer drug improves therapeutic response in animal model of human epithelial cancer. Cancer Research 65(12): 5317-5324.

32. Malik N, Wiwattanapatapee R, Klopsch R, Lorenz K, Frey H, et al. (2000) Dendrimers: Relationship between structure and biocompatibility in vitro, and preliminary studies on the biodistribution of 125I-labelled polyamidoamine dendrimers in vivo. Journal of Controlled Release 65(12): 133-148.

33. Padilla De Jesus OL, Ihre HR, Gagne L, Frechet JM, Szoka FC (2002) Polyester dendritic systems for drug delivery applications: In vitro and in vivo evaluation. Bioconjugate Chemistry 13(3): 453-461.

34. Sarin H, Kanevsky AS, Fung SH, Butman J, Cox R, et al. (2009) Metabolically stable bradykinin B2 receptor agonists enhance transvascular drug delivery into malignant brain tumors by increasing drug half-life. Journal of Translational Medicine 7: 33.

35. Sarin H (2010) Physiologic upper limits of pore size of different blood capillary types and another perspective on the dual pore theory of microvascular permeability. Journal of Angiogenesis Research 2: 14.

36. Huang Y, Coman D, Ali MM, Hyder F (2015) Lanthanide ion (III) complexes of $1,4,7,10$ - tetraazacyclododecane-1,4,7,10-tetraaminophosphonate for dual biosensing of $\mathrm{pH}$ with Chemical Exchange Saturation Transfer (CEST) and Biosensor Imaging of Redundant Deviation in Shifts (BIRDS). Contrast Media \& Molecular Imaging 10(1): 51-58.

37. Coman D, Kiefer GE, Rothman DL, Sherry AD, Hyder F (2011) A lanthanide complex with dual biosensing properties: CEST (Chemical Exchange Saturation Transfer) and BIRDS (biosensor Imaging of redundant Deviation in Shifts) with europium DOTA-tetraglycinate. NMR in Biomedicine 24(10): 1216-1225.
38. Coman D, de Graaf RA, Rothman DL, Hyder F (2013) In vivo threedimensional molecular imaging with Biosensor Imaging of Redundant Deviation in Shifts (BIRDS) at high spatiotemporal resolution. NMR in Biomedicine 26(11): 1589-1595.

39. Kobayashi H, Brechbiel MW (2003) Dendrimer-based macromolecular MRI contrast agents: characteristics and application. Molecular Imaging 2(1): 1-10.

40. Sato N, Kobayashi H, Hiraga A, Saga T, Konishi J, et al. (2001) Pharmacokinetics and enhancement patterns of macromolecular MR contrast agents with various sizes of polyamidoamine dendrimer cores. Magnetic Resonance in Medicine 46(6): 1169-1173.

41. Sarin H (2009) Recent progress towards development of effective systemic chemotherapy for the treatment of malignant brain tumors. Journal of Translational Medicine 7: 77.

42. Sonavane G, Tomoda K, Makino K (2008) Biodistribution of colloidal gold nanoparticles after intravenous administration: effect of particle size. Colloids and Surfaces B, Biointerfaces 66(2): 274-280.

43. Binion DG, Otterson MF, Rafiee P (2008) Curcumin inhibits VEGFmediated angiogenesis in human intestinal microvascular endothelial cells through COX-2 and MAPK inhibition. Gut 57(11): 1509-1517.

44. Sarin H (2010) Overcoming the challenges in the effective delivery of chemotherapies to CNS solid tumors. Therapeutic Delivery 1(2): 289305.

45. Grobner T (2006) Gadolinium-a specific trigger for the development of nephrogenic fibrosing dermopathy and nephrogenic systemic fibrosis? Nephrology, Dialysis, Transplantation 21(4): 1104-1108.

46. Marckmann P, Skov L, Rossen K, Dupont A, Damholt M, et al. (2006) Nephrogenic systemic fibrosis: suspected causative role of gadodiamide used for contrast-enhanced magnetic resonance imaging. Journal of the American Society of Nephrology 17(9): 2359-2362.

47. Kurtkoti J, Snow T, Hiremagalur B (2008) Gadolinium and nephrogenic systemic fibrosis: Association or causation. Nephrology (Carlton) 13(3): 235-241.

48. Kanda T, Ishii K, Kawaguchi H, Kitajima K, Takenaka D (2014) High signal intensity in the dentate nucleus and globus pallidus on unenhanced T1weighted MR images: Relationship with increasing cumulative dose of a gadolinium-based contrast material. Radiology 270(3): 834-841.

49. Kanda T, Fukusato T, Matsuda M, Toyoda K, Oba H, et al. (2015) Gadolinium-based contrast agent accumulates in the brain even in subjects without severe renal dysfunction: Evaluation of autopsy brain specimens with inductively coupled plasma mass spectroscopy. Radiology 276(1): 228-232.

50. Chang CY, Cheng TJ, Chang FR, Wang HY, Kan WC, et al. (2011) Macrophage mediated anti-proliferation effects of Anthodia camphorata nonpolysaccharide-based extracts on human hepatoma cells. Bioscience, Biotechnology, and Biochemistry 75(4): 624-632.

51. Cirstoiu-Hapca A, Bossy-Nobs L, Buchegger F, Gurny R, Delie F (2007) Differential tumor cell targeting of anti-HER2 (Herceptin) and antiCD20 (Mabthera) coupled nanoparticles. International Journal of Pharmaceutics 331(2): 190-196.

52. Rapoport N, Gao Z, Kennedy A (2007) Multifunctional nanoparticles for combining ultrasonic tumor imaging and targeted chemotherapy. Journal of the National Cancer Institute 99(14): 1095-1106.

53. Ponce AM, Vujaskovic Z, Yuan F, Needham D, Dewhirst MW (2006) Hyperthermia mediated liposomal drug delivery. International Journal of Hyperthermia 22(3): 205-213.

54. Ali MM, Woods M, Suh EH, Kovacs , Tircso G, et al. (2007) Albuminbinding PARACEST agents. Journal of Biological Inorganic Chemistry 12(6): 855-865. 
55. Ahn BH, Lee JH, Bae YS (2003) Identification of mutations in protein kinase CKIIbeta subunit that affect its binding to ribosomal protein L41 and homodimerization. Journal of Biochemistry and Molecular Biology 36(4): 344-348.

56. Han L, Huang R, Li J, Liu S, Huang S, et al. (2011) Plasmid pORFhTRAIL and doxorubicin co- delivery targeting to tumor using peptideconjugated polyamidoamine dendrimer. Biomaterials 32(4): 1242-1252.

57. Li Y, He H, Jia X, Lu WL, Lou J (2012) A dual targeting nanocarrier based on poly(amidoamine) dendrimers conjugated with transferrin and tamoxifen for treating brain gliomas. Biomaterials 33(15): 3899-3908.

58. Cho YA, Lee W, Choi JS (2012) Effects of curcumin on the pharmacokinetics of tamoxifen and its active metabolite, 4-hydroxytamoxifen, in rats: possible role of $\mathrm{CYP}_{3} \mathrm{~A}_{4}$ and P-glycoprotein inhibition by curcumin. Die Pharmazie 67(2): 124-130.

59. Choi BH, Kim CG, Bae YS, Lim Y, Lee YH, et al. (2008) p21 Waf1/Cip1 expression by curcumin in U-8 $\mathrm{MG}$ human glioma cells: Role of early growth response-1 expression. Cancer Research 68(5): 1369-1377.

60. Choi BH, Kim CG, Lim Y, Shin SY, Lee YH (2008) Curcumin down-regulates the multidrug- resistance mdr1b gene by inhibiting the PI3K/Akt/NF kappa B pathway. Cancer Letters 259(1): 111-118.

61. Choi H, Chun YS, Kim SW, Kim MS, Park JW (2006) Curcumin inhibits hypoxia-inducible factor- 1 by degrading aryl hydrocarbon receptor nuclear translocator: A mechanism of tumor growth inhibition. Molecular Pharmacology 70(5): 1664-1671.

62. Choudhuri T, Pal S, Das T, Sa G (2005) Curcumin selectively induces apoptosis in deregulated cyclin D1-expressed cells at G2 phase of cell cycle in a p53-dependent manner. The Journal of Biological Chemistry 280(20): 20059-20068.

63. Yallapu MM, Jaggi M, Chauhan SC (2012) Curcumin nanoformulations: A future nanomedicine for cancer. Drug Discovery Today 17(1-2): 71-80.
64. Yang CL, Liu YY, Ma YG, Xue Y, Liu D, et al. (2012) Curcumin blocks small cell lung cancer cells migration, invasion, angiogenesis, cell cycle and neoplasia through Janus kinase-STAT3 signalling pathway. PloS One 7(5): e37960.

65. Yang CW, Chang CL, Lee HC, Chi CW, Pan JP, et al. (2012) Curcumin induces the apoptosis of human monocytic leukemia THP-1 cells via the activation of JNK/ERK pathways. BMC Complementary and Alternative Medicine12: 22.

66. Senft C, Polacin M, Priester M, Seifert V, Kogel D, et al. (2010) The nontoxic natural compound Curcumin exerts anti-proliferative, antimigratory, and anti-invasive properties against malignant gliomas. BMC Cancer 10: 491.

67. Yallapu MM, Othman SF, Curtis ET, Bauer N, Neeraj C, et al. (2012) Curcumin-loaded magnetic nanoparticles for breast cancer therapeutics and imaging applications. International Journal of Nanomedicine 7: 1761-1779.

68. Ravindranath V, Chandrasekhara N (1980) Absorption and tissue distribution of curcumin in rats. Toxicology 16(3): 259-265.

69. Ali MM, Liu G, Shah T, Flask CA, Pagel MD (2009) Using two chemical exchange saturation transfer magnetic resonance imaging contrast agents for molecular imaging studies. Accounts of Chemical Research 42(7): 915-924.

70. Ali MM, Janic B, Babajani-Feremi A, Varma RS, Iskander ASM, et al. (2010) Changes in vascular permeability and expression of different angiogenic factors following anti-angiogenic treatment in rat glioma. PloS One 5(1): e8727.

71. Burgos A, Kaplan R, Rodríguez N, Vetanzo M, Morelatto R, et al. (2008) Malignant melanoma of the oral cavity. Rev Fac Cien Med Univ Nac Cordoba 65(2): 70-73. 